\title{
An Anthropometric and Three-Dimensional Computed Tomographic Evaluation of Two-Point Fixation of Zygomatic Complex Fractures
}

\author{
Taehee Jo, Junhyung Kim \\ Department of Plastic and Reconstructive Surgery, Keimyung University Dongsan Medical Center, Daegu, Korea
}

Background Maintaining stability and restoring the aesthetic appearance are the fundamental goals when managing zygomatic fractures. We aimed to evaluate the stability and anthropometric outcomes of zygomatic fracture patients who underwent two-point fixation involving the infraorbital rim and zygomaticomaxillary buttress via the transconjunctival and gingivobuccal approaches without any skin incisions.

Methods We examined 15 zygomatic fracture patients who underwent two-point fixation during a 3-year period. Stability was evaluated using three-dimensional facial bone computed tomography. Superoinferior and anteroposterior displacement of the zygoma was quantified. The aesthetic appearance of the periorbital region was evaluated using indirect anthropometry with standardized clinical photographs. The ratios between the eye fissure height and width, and lower iris coverage ratio were used to evaluate aesthetical changes. The bony displacement and aesthetic ratios were analyzed using Wilcoxon or Friedman tests. The correlation between the preoperative zygoma position and anthropometric values was analyzed.

Results The positions of the zygoma were similar to those on the contralateral side at the long-term follow-up. The preoperative anthropometric measurements on the fractured side differed from those on the contralateral side, although these values were close to the normal values at the long-term follow-up. Furthermore, we noted that the anteroposterior displacement strongly positively correlated with the lower iris coverage rate (Spearman's coefficient $=0.678, P=0.005$ ).

Conclusions Two-point fixation of zygomatic fractures achieved stable outcomes on longterm follow-up, and also appeared to be reliable in restoring the aesthetic appearance of the periorbital region.

Keywords Zygomatic fractures / Fracture fixation / Anthropometry
Correspondence: Junhyung Kim Department of Plastic and Reconstructive Surgery, Keimyung University School of Medicine, 56 Dalseong-ro, Jung-gu, Daegu 700-712, Korea

Tel: +82-53-250-7635

Fax: +82-53-255-0632

E-mail: med69@dsmc.or.kr
This article was presented at the 71st congress of Korean society of plastic and reconstructive surgeons, Nov 1-3, 2013, in Seoul, Korea.

No potential conflict of interest relevant to this article was reported.

Received: 28 Mar 2014 • Revised: 24 Jun 2014 • Accepted: 8 Jul 2014

pISSN: 2234-6163 • elSSN: 2234-6171 • http://dx.doi.org/10.5999/aps.2014.41.5.493 • Arch Plast Surg 2014;41:493-499

\section{INTRODUCTION}

The primary goals in the treatment of zygomatic fractures include the restoration of the projection and the height of the zygoma by accurate reduction and the restoration of the aesthetic appearance. Adequate exposure and reduction by multiple incisions and strong fixation by plates are believed to be essential to achieving satisfactory results. However, these conventional approaches require a long operation time and may lead to unnecessary scarring. To overcome these limitations, several new at- 
tempts have been made in the management of zygomatic fractures $[1,2]$.

Previous studies on the appearance of zygomatic fractures were limited to reporting the rates of gross complications such as lower lid retractions, ectropions, and entropions. Smaller changes to the periorbital structures were neglected. Moreover, previous studies on the stability of zygomatic fractures primarily involved experiments on cadaveric heads $[3,4]$, and did not correspond with the actual clinical situations $[5,6]$. The recent widespread use of three-dimensional computed tomography (3D CT) made it easier to verify the position and displacement of the zygomatic body in vivo. Consequently, less aggressive methods to obtain stability were developed $[7,8]$.

Recently, the main concern of patients who experience zygomatic fractures is whether they can recover an aesthetically pleasing appearance. To minimize scar formation, our institution has been performing two-point fixations involving the infraorbital rim and the zygomaticomaxillary buttress for zygomatic fractures via the transconjunctival and gingivobuccal incisions. In the present study, we aimed to evaluate the stability of the zygoma with 3D CT and to evaluate the periorbital anthropometric changes using standardized photographs in patients who underwent zygomatic fracture surgeries with the two-point fixation technique.

\section{METHODS}

After institutional review board approval, our study included patients who underwent surgical repair of zygomatic fractures at our institution between January 2009 and December 2012. Two-point fixation of the fractures was accomplished via transconjunctival and gingivobuccal incisions. Preoperative and 6-month postoperative 3D CT images were obtained in all patients. Under general anesthesia, the infraorbital rim was exposed via a preseptal approach after a transconjunctival incision. The fracture was fixed using 0.4-mm-thick plates (micro plate, Matrix midface plate, Synthes CMF, West Chester, PA, USA). In patients with associated orbital floor fractures, a porous polyethylene implant (SynPOR, Synthes CMF) was placed after reduction [9]. The zygomaticomaxillary buttress was exposed by gingivobuccal incision, and fixed using a $0.5-\mathrm{mm}$-thick plate (mini plate, Matrix midface plate, Synthes CMF) after adequate reduction. Preoperative 3D CT and standardized photographs were obtained for every patient. At the 6-month follow-up, 3D CT was performed and standardized photographs were retaken after consent was obtained from the patients.

After performing maxillofacial tomography scans, the 3D CT images were reconstructed by using three-dimensional recon- struction software (Definition Flash, Siemens Healthcare, Forchheim, Germany). The craniofacial structures in the threedimensional images were reoriented according to the reference planes - the Frankfurt horizontal plane and the infraorbital rims.

Frontal view photographs were obtained with a DSLR camera (D300, Nikon, Tokyo, Japan). Patients were consistently positioned according to the Frankfurt line and the intercanthal axis using the cephalostat [10]. Photographic analysis was performed using the Adobe Photoshop CS2 (Adobe Inc., San Jose, CA, USA) measurement software tool by a single plastic surgeon.

The height and the projection of the zygoma complex were evaluated on preoperative and postoperative 3D CT images. The height was assessed using the frontal view images and was quantified as the vertical difference (VD) ratio of the vertical distance from the supraorbital rim to the infraorbital rim on the fracture side compared to that on the contralateral side (Fig. 1). When the fractured zygoma is inferiorly displaced, the VD ratio would be greater than 1 . The projection of the zygoma was also evaluated in the axial view images and was quantified as the horizontal difference (HD) ratio of the distance between the anterior margin and the posterior margin of the fossa temporalis on the fractured side compared to that on the contralateral side (Fig. 2). If the fractured zygoma was posteriorly displaced, the HD value would be less than 1 .

The eye fissure index (EFI) and lower iris coverage index (LIC) were estimated for both the fractured and the contralateral sides using the frontal view of standardized photographs. EFI was defined as the ratio of eye fissure width (EFW) to eye fissure height $(\mathrm{EFH})$. LIC was defined as the percentage of the

\section{Fig. 1. Vertical difference on frontal view}

The line connecting the supraorbital rim margins on the fractured and on the contralateral side was defined as the horizontal base line. The lowermost point of the infraorbital rim margin was set as point $A$ on the contralateral side, and the distance from the base line was defined as distance a. On the fractured side, these components were defined as point $B$ and distance $b$, respectively. The vertical difference was defined as the ratio of $b$ to a ( $b / a)$.

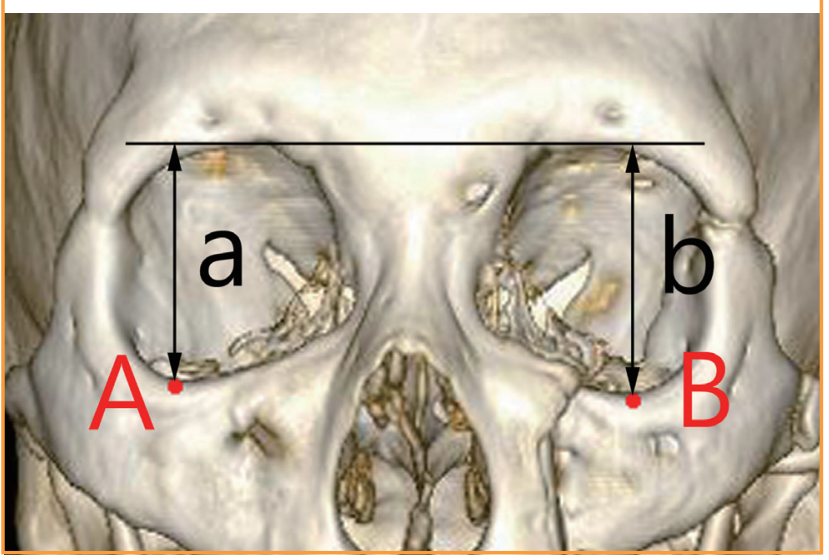


iris that was covered by the lower eyelid (Fig. 3).

To evaluate the inter-rater reliability of indirect anthropometry by photogrammetric analysis, we assessed 5 additional patients with zygomatic fractures, before they underwent surgery. Two plastic surgery residents individually photographed the patients, and calculated the EFI and LIC of the fractured and contralateral sides. The measurements were analyzed by intra-class correlation coefficient for interobserver reliability.

To analyze the changes in VD and HD, nonparametric Wil-

\section{Fig. 2. Horizontal difference on axial view}

The line connecting the posterior margins of the fossa temporalis on the fractured and the contralateral sides was defined as the horizontal base line. The most distant point on the anterior margin was considered as point A on the contralateral side, and the distance from the base line was defined as distance a. On the fractured side, these components were defined as point $B$ and distance $b$, respectively. The horizontal difference was defined as the ratio of $b$ to a (b/a).

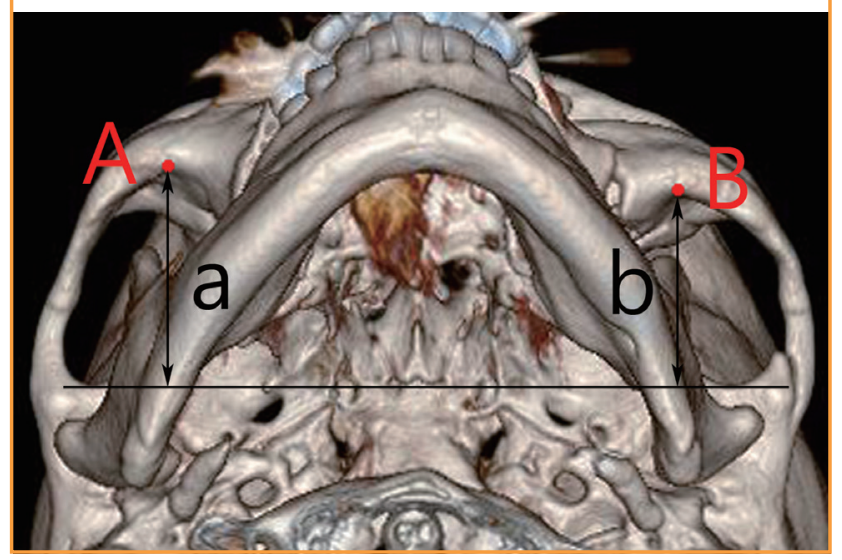

coxon test was conducted using the PASW (ver. 18.0, SPSS Inc., Chicago, IL, USA) for Windows. For EFI and LIC, Friedman test was conducted. Moreover, to determine the correlation between the preoperative values of VD and $\mathrm{HD}$ and the preoperative values of EFI and LIC, correlation analysis was performed using the Spearman's coefficient value as the correlation coefficient. A P-value of $<0.05$ was considered to be statistically significant. Data are reported as medians and ranges.

\section{RESULTS}

\section{Subjects}

We included 15 patients (male, 9; female, 6) in the present study. The median age of subjects was 33.5 years (range, 19-64 years).

\section{Measurement reliability}

In total, 10 eyes in 5 subjects were analyzed to evaluate the measurement reliability of the photogrammetry analysis. The intraclass correlation coefficient was 0.969 for EFI and 0.981 for LIC $(\mathrm{P}<0.05)$. Good reliability was observed for both these measurements.

\section{Outcomes}

Both the height and the projection on the fractured side were restored to a point close to that on the contralateral side. The VD value was 1.019 (1.000-1.104) preoperatively and was restored to $1.003(0.987-1.010)$ at the 6-month follow-up examination $(\mathrm{P}=0.002)$. The HD value was $0.912(0.841-0.973)$

\section{Fig. 3. Diagram indicating the EFI and LIC}

(A) Eye fissure index (EFI) was defined as the ratio of the eye fissure width (EFW) to the eye fissure height $(E F H)(E F I=E F W / E F H)$. EFH was defined as the distance from the margin of the superior palpebra to the margin of the inferior palpebral (Ps-Pi), whereas EFW was defined as the distance from the medial and lateral margin. Moreover, lower iris coverage index (LIC) was defined as the percentage of the iris that was covered by the lower eyelid. The distance from the center point of the iris to the lower eyelid margin (lc-Pi) is distracted from the radius to calculate the length covered by the eyelid. This value is then divided by the total iris diameter (ID) to yield the LIC value (LIC= [ID/2-(Ic-Pi)]/ID). (B) Partial close-up image of the periorbital region on a standardized photograph.

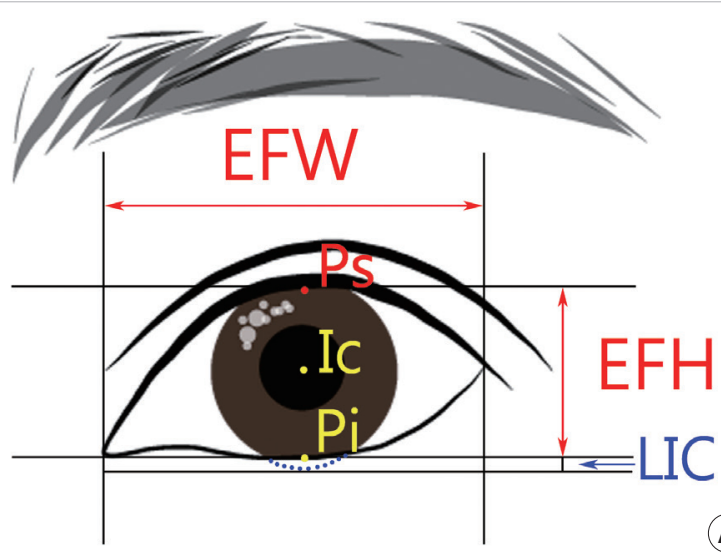

(A)

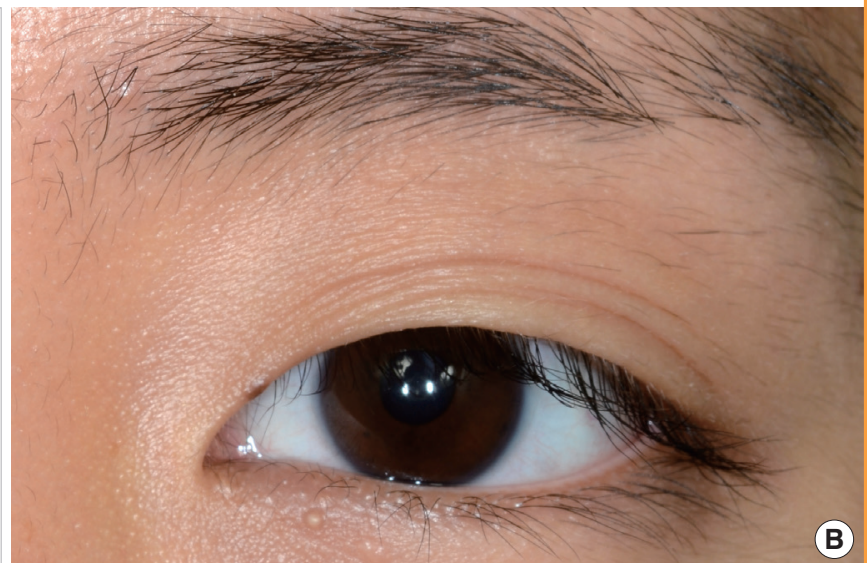


Table 1. Eye fissure index and lower iris coverage index values on standardized photograph

\begin{tabular}{|c|c|c|c|c|}
\hline \multirow{2}{*}{ Characteristic } & \multirow{2}{*}{ Contralateral } & \multicolumn{2}{|c|}{ Fractured } & \multirow{2}{*}{ P-value ${ }^{a)}$} \\
\hline & & Preoperatively & Postoperative 6 mo later & \\
\hline \multicolumn{5}{|l|}{ Eye fissure index } \\
\hline Median & 0.350 & 0.361 & 0.340 & 0.192 \\
\hline Range & $0.316-0.434$ & $0.310-0.366$ & $0.310-0.432$ & - \\
\hline \multicolumn{5}{|c|}{ Lower iris coverage index } \\
\hline Median & 0.035 & 0.025 & 0.036 & 0.132 \\
\hline Range & $-0.082-0.098$ & $-0.082-0.080$ & $-0.082-0.090$ & - \\
\hline
\end{tabular}

Table 2. Correlation analysis for VD, HD, EFI, and LIC

\begin{tabular}{|lll|}
\hline Characteristic & EFI & LIC \\
\hline VD & & \\
Spearman's coefficients & -0.426 & -0.090 \\
P-value & 0.113 & 0.749 \\
Fisher's 95\% Cl & $-0.777-0.126$ & $-0.587-0.456$ \\
HD & & \\
Spearman's coefficients & 0.269 & 0.678 \\
P-value & 0.332 & 0.005 \\
Fisher's 95\% Cl & $-0.298-0.695$ & $0.239-0.887$ \\
\hline
\end{tabular}

VD, vertical difference; HD, horizontal difference; EFI, eye fissure index; LIC, lower iris coverage index; $\mathrm{Cl}$, confidence interval.

preoperatively, and was restored to $0.989(0.950-1.002)$ at the 6-month follow-up examination $(\mathrm{P}=0.001)$.

Although not statistically significant, the EFI on the fractured side did not greatly differ from that on the contralateral side before the surgery but the difference between the EFI on the fractured side and that on the contralateral side was slightly lower at the final follow-up examination $(\mathrm{P}=0.192)$. The LIC on the fractured side preoperatively was somewhat different to that on the contralateral side. However, at the 6-month follow-up examination, the LIC was restored to a value close to the initial value on the contralateral side $(\mathrm{P}=0.132)$ (Table 1$)$.

The VD preoperatively was not significantly correlated with the EFI or LIC. Moreover, the HD did not significantly correlate with the EFI; however, the HD indicted a strongly significant positive correlation with the LIC (Table 2, Fig. 4).

\section{Case}

A 32-year-old woman who experienced a zygomatic fracture on the left side underwent two-point fixation. The preoperative CT image indicated inferior and posterior dislocation of the zygoma body along with small zygomaticofrontal suture diastasis. At 6 months postoperatively, $\mathrm{CT}$ images indicated that the zygoma was restored to a point adjacent to the normal position (Fig. 5). Although periorbital swelling was observed preoperatively, the patient indicated that the aesthetic appearance of the periorbital

\section{Fig. 4. Scatter diagram of HD, EFI, and LIC}

(A) EFI was not significantly correlated with HD. (B) LIC showed a strong significant association with HD. HD, horizontal difference; $\mathrm{EFI}$, eye fissure index; LIC, lower iris coverage index.
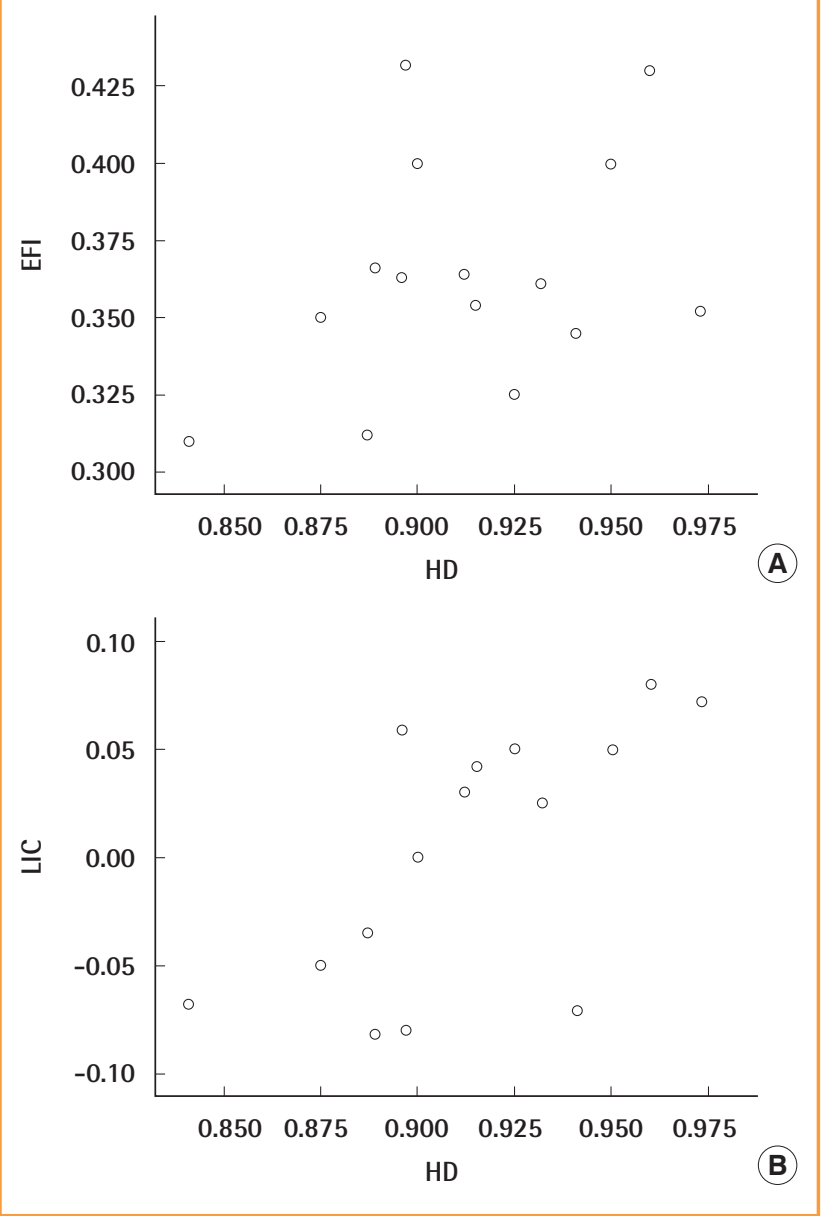

region was satisfactory at the long-term follow-up (Fig. 6).

\section{DISCUSSION}

Zygomatic fractures have traditionally been treated using 3-point fixations involving the zygomaticomaxillary buttress, infraorbital rim, and zygomaticofrontal suture [3]. Rohner et al. 
Fig. 5. Three-dimensional computed tomography of the patient case

(A) Preoperative frontal view showing inferior dislocation of the zygoma body. (B) Preoperative coronal view showing posterior dislocation of the zygoma body. (C) Postoperative frontal view with a microplate on the infraorbital rim and a miniplate on the zygomaticomaxillary buttress. (D) Postoperative coronal view demonstrating restored projection of the zygoma body.
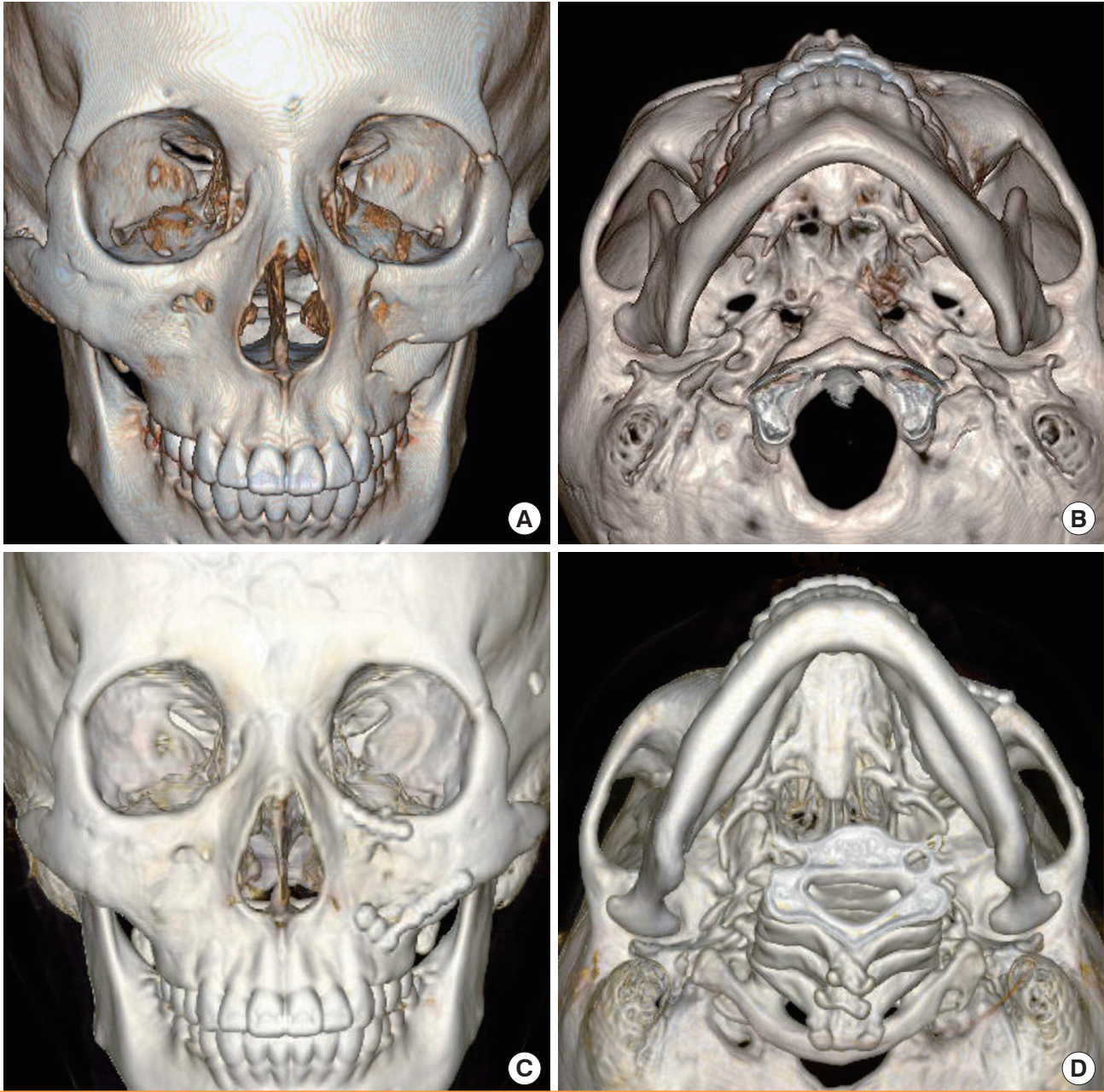

Fig. 6. Standardized photograph of the patient case

(A) Preoperative photograph showing slight swelling at the left eye. (B) Postoperative photograph showing good restoration and satisfactory results.

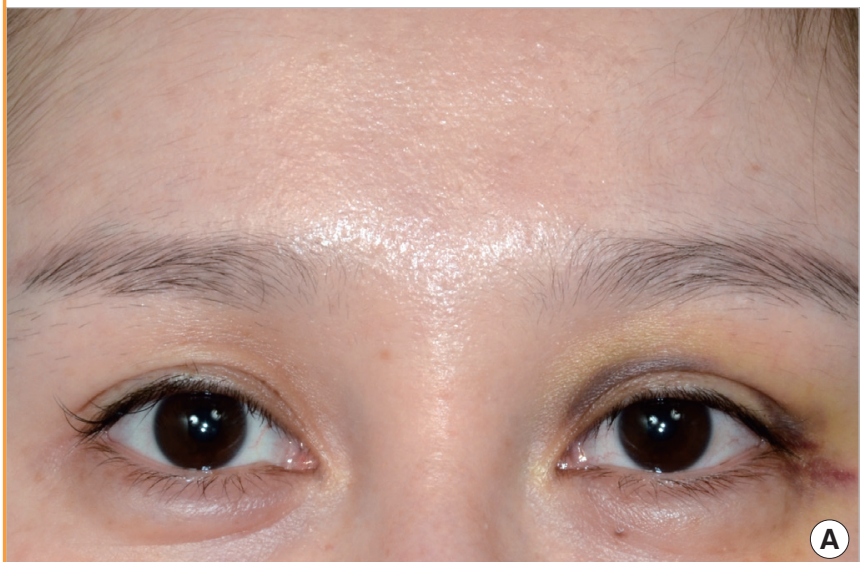

[11] suggested that fixation at the zygomaticofrontal suture is the most important factor for achieving stability. Rinehart et al. [3] indicated that 3-point fixation using miniplates on the zygo-

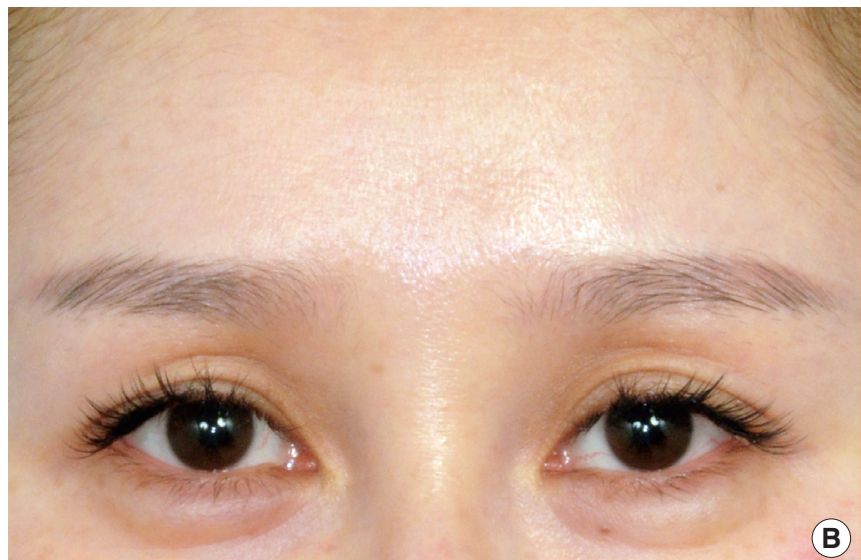

maticomaxillary buttress, infraorbital rim, and zygomaticofrontal suture is essential for achieving stability. However, these previous studies reported the outcomes of experimental studies on 
cadaveric heads. The experiments were performed on bare skulls, after making deliberate fractures and artificially inducing the expected downward acting masseteric muscle forces. However, Dal Santo et al. [5] reported that the actual masseteric muscle force that pulls down the zygoma is substantially reduced for 4 weeks after the trauma. Furthermore, Tarabichi [6] indicated that the zygoma is covered with soft tissue, such as the supereficial musculo-aponeurotic system, which naturally acts as a resistant force against the masseteric downward forces. Attempts have been made to avoid transcutaneous incisions for zygomaticofrontal sutures, such as upper eyelid transconjunctival incisions; however, these approaches have not been generally accepted [12]. Certain surgeons state that the zygomaticofrontal suture is the least important point for determining the degree of rotation of the fracture, and the visualization with this approach is also not very helpful for achieving accurate reduction [13]. In the present study, we performed zygomatic fracture reduction via the transconjunctival and gingivobuccal approaches, without exposure or fixation at the zygomaticofrontal suture. The long-term stability was evaluated at preoperative and postoperative 3D CT examinations, and the periorbital appearance was evaluated by indirect anthropometry using standardized photogrammetry.

The displacement and stability of the fracture were evaluated using quantified indices, including the superoinferior and anteroposterior displacement. Zygomatic fractures generally result in inferior and posterior displacement of the orbital rim. The VD represents the degree of inferior displacement of the zygoma. Preoperatively, we observed that the zygoma was inferiorly displaced by $2 \%$ and the values were not evenly distributed; the standard deviation was quite large. At the long-term follow-up examination, the displacement was restored to a point close to the normal value, and the values were evenly distributed; the standard deviation was quite small. The HD represents the degree of posterior displacement. The average degree of posterior displacement of the zygoma was approximately $10 \%$. At the long-term follow-up examination, this was also restored to a value close to the normal value. This method for determining the zygomatic displacement is a modification of the method suggested by Fujioka et al. [14], who estimated the projection and the height by measuring the changes in the angle of the zygoma; however, when we performed this method, we observed that it lacked reproducibility. Moreover, when calculating the VD value, some patients exhibited a bilateral or unilateral supraorbital notch, which is a variation of the supraorbital foramen. In order to accurately outline the orbital rim, we ignored these notches during the measurements.

An important advantage of photogrammetry over direct an- thropometry involves the ease of assessment (photographs are easier to analyze than evaluation of the patients face); however, its disadvantage is the fact that it is an indirect type of anthropometry. However, obtaining standardized photographs and comparing the ratios enables the evaluation of changes and differences between the fractured and contralateral sides before and after the operations [15]. Even small aesthetic changes in the periorbital region can result in changes in the facial expression and impression. Based on this finding, it was suggested that periorbital anthropometry, which detects small changes, could serve as a better method for evaluating zygomatic fractures than the measurement of bony changes. The inferior and posterior displacement of the orbital rim affects the orbital septum, which is attached to the orbital rim; this consequently results in the downward pulling of the lower eyelid. EFI is the ratio between $\mathrm{EFH}$ and eye fissure width, whereas LIC is the ratio between the coverage of the iris by the lower eyelid to the iris diameter. Lower eyelid retraction could cause changes in the EFI and LIC values. Moreover, LIC is known to play a decisive role in the perioribtal appearance [16]. In addition, Raschke et al. [17] indicated that different approaches may yield varying changes in the perioribtal anthropometry parameters; they found that the changes in EFI and LIC in such cases were statistically significant. In the present study, the EFI on the fracture side was not greatly different from that on the contralateral side prior to reduction. Moreover, the LIC values were negative after the trauma. At the longterm follow-up examination, the EFI and LIC were restored to the normal values, indicating that the reduction and fixation were stable and the aesthetic appearance of the periorbital region was suitable.

To evaluate the reliability of indirect anthropometry of the periorbital region, we selected 5 additional patients with zygomatic fractures. Two plastic surgeons who had at least 2 years of experience in clinical photography individually obtained images of the patients, and estimated the anthropometric measurements. The pictures were repeatedly obtained until the photographer was satisfied; the images were taken in a studio with the help of a cephalostat, which maintains the patients in a consistent position [10]. We examined both the fractured and contralateral sides, and thus, 10 eyes were evaluated. Both the EFI and the LIC demonstrated good reliability. As the standardized photographs of all the patients in the study, including the 15 study patients, were taken in the studio using this method, we believe that reliability may not be a problem in the present study.

Assessing the relationship between the underlying bony structure and the aesthetics of the overlying periorbital region is difficult because periorbital anthropometry in the acute traumatic phase is usually affected by soft tissue changes, such as an edema 
or hematoma. Moreover, the EFI values appear to be influenced by soft tissue changes. In the present study, the fractured and the contralateral sides did not exhibit significant differences, even when the fracture was present. However, the LIC on the fractured side was different from that on the contralateral side. The VD value preoperatively was not associated with the EFI or LIC values. However, the $\mathrm{HD}$ value indicated a positive association with the EFI and the LIC values. This indicates that superoinferior displacement of the zygoma would not cause meaningful changes to the appearance of the eyes, although anteroposterior displacement would cause a meaningful change. In particular, the LIC value is relatively strongly correlated with the HD value. This indicates that LIC measurement could be used to indirectly determine the stability of the zygoma.

The presence of diastasis in the zygomaticofrontal suture plays an important role when determining whether this portion should be exposed. Surgeons generally prefer to perform accurate reduction under full exposure with a lateral brow incision. All the patients in the present study had zygomaticofrontal suture displacement, although the displacement was not very severe. Therefore, further studies involving patients with severe displacement would help assess whether the current approach has a broader application in the treatment of zygomatic fractures.

A few surgeons state that one-point fixation at the zygomaticomaxillary buttress is sufficient [14]. However, the assessment of the orbital floor by using a transconjunctival approach is essential for repairing associated blow-out fractures, as the orbital floor occasionally cannot be appropriately assessed on facial bone CT. Exposure of the orbital structure also helps in identifying the alignment of the greater wing of the sphenoid bone.

Even when considering the limitations of the technique, we believe that the treatment of zygomatic fractures by using transconjunctival and gingivobuccal incisions is advantageous in that it does not result in any incision scars and achieves favorable and stable anatomic and anthropometric outcomes. This approach could serve as a novel alternative for socially active young male or female patients who are sensitive to aesthetic changes.

\section{REFERENCES}

1. Pospisil OA, Fernando TD. Review of the lower blepharoplasty incision as a surgical approach to zygomatic-orbital fractures. Br J Oral Maxillofac Surg 1984;22:261-8.

2. Manson PN, Ruas E, Iliff N, et al. Single eyelid incision for exposure of the zygomatic bone and orbital reconstruction. Plast Reconstr Surg 1987;79:120-6.

3. Rinehart GC, Marsh JL, Hemmer KM, et al. Internal fixation of malar fractures: an experimental biophysical study.
Plast Reconstr Surg 1989;84:21-5.

4. O'Hara DE, DelVecchio DA, Bartlett SP, et al. The role of microfixation in malar fractures: a quantitative biophysical study. Plast Reconstr Surg 1996;97:345-50.

5. Dal Santo F, Ellis E 3rd, Throckmorton GS. The effects of zygomatic complex fracture on masseteric muscle force. J Oral Maxillofac Surg 1992;50:791-9.

6. Tarabichi M. Transsinus reduction and one-point fixation of malar fractures. Arch Otolaryngol Head Neck Surg 1994; 120:620-5.

7. Kim JH, Lee JH, Hong SM, et al. The effectiveness of 1-point fixation for zygomaticomaxillary complex fractures. Arch Otolaryngol Head Neck Surg 2012;138:828-32.

8. Hwang K. One-point fixation of tripod fractures of zygoma through a lateral brow incision. J Craniofac Surg 2010;21: 1042-4.

9. Novelli G, Ferrari L, Sozzi D, et al. Transconjunctival approach in orbital traumatology: a review of 56 cases. J Craniomaxillofac Surg 2011;39:266-70.

10. Kwon HJ, Han KH, Kim JH, et al. Photogrammetry based on standardized clinical photography using cephalostat: comparison with anthropometric analysis. J Korean Soc Plast Reconstr Surg 2007;34:24-36.

11. Rohner D, Tay A, Meng CS, et al. The sphenozygomatic suture as a key site for osteosynthesis of the orbitozygomatic complex in panfacial fractures: a biomechanical study in human cadavers based on clinical practice. Plast Reconstr Surg 2002;110:1463-71.

12. Langsdon PR, Rohman GT, Hixson R, et al. Upper lid transconjunctival versus transcutaneous approach for fracture repair of the lateral orbital rim. Ann Plast Surg 2010;65: 52-5.

13. Kelley P, Hopper R, Gruss J. Evaluation and treatment of zygomatic fractures. Plast Reconstr Surg 2007;120:5S-15S.

14. Fujioka M, Yamanoto T, Miyazato O, et al. Stability of oneplate fixation for zygomatic bone fracture. Plast Reconstr Surg 2002;109:817-8.

15. Han K, Kim D, Park M, et al. Web uni-limb z-plasty for correction of alar web deformity in unilateral cleft lip nasal deformities: photogrammetric analysis. J Korean Soc Plast Reconstr Surg 2011;38:740-6.

16. Starck WJ, Griffin JE Jr, Epker BN. Objective evaluation of the eyelids and eyebrows after blepharoplasty. J Oral Maxillofac Surg 1996;54:297-302.

17. Raschke GF, Rieger UM, Bader RD, et al. The zygomaticomaxillary complex fracture-an anthropometric appraisal of surgical outcomes. J Craniomaxillofac Surg 2013;41:331-7. 